\title{
Stakeholderanalyse in der Planung ärztlicher Weiterbildungs-Curricula
}

\section{Roman Hari}

Dr. med., Projektleiter Weiterbildung SOS Ärzte

\begin{abstract}
Das Weiterbildungsangbot hat sich aufgrund des Ärztemangels zu einem wichtigen Kriterium bei der Stellenwahl junger Ärzte entwickelt. In der Planung eines Weiterbildungs-Curriculums ist die gezielte Erfragung der Bedürfnisse wichtiger Stakeholder ein Kernpunkt. Hierfür wird ein systematischer Ansatz aus dem Bereich Projektmanagement vorgestellt und am Beispiel der SOS Ärzte Zürich erläutert.
\end{abstract}

\section{Weiterbildung als Rekrutierungsfaktor}

Der Ärztemangel in der Schweiz führt seit einigen Jahren in verschiedenen Fachgebieten zur neuartigen Situation, dass Assistenzärzte zwischen mehreren Stellen auswählen können [1]*. Weiterbildungsstätten müssen daher gezielt Anreize schaffen, um im Werben um die vorhandenen Arbeitskräfte konkurrenzfähig zu bleiben. Das angebotene Weiterbildungsprogramm wird dabei zu einem der wichtigsten Auswahlkriterien, wie eine Zürcher Studie mit Staatsexamensabsolventen kürzlich zeigte [2]. Somit wird die Weiterbildung direkt oder indirekt zum Rekrutierungsfaktor und zu einem Ansatzpunkt gezielter betriebswirtschaftlicher Investitionen.

verwendete männlich Form gilt für Personen beiderlei Geschlechts.

* Die Literaturangaben finden sich unter www.saez.ch $\rightarrow$ Aktuelle Ausgabe oder Archiv $\rightarrow 2 \mathrm{O} 15 \rightarrow 2 \mathrm{O} / 21$.

\section{Neuausrichtung der Weiterbildung}

Um dieser neuen Rolle der Weiterbildung gerecht zu werden und um die potentiell neu geschaffenen finanziellen und personellen Ressourcen längerfristig im Sinne der Weiterbildung zu sichern, ist aus der Per-
Analyse des groupes d'intérêts dans la planification des cursus de formation postgraduée médicale

La pénurie croissante de médecins oblige à reconsidérer la formation postgraduée sous l'angle économique en tant que facteur essentiel du recrutement. Pour ne pas manquer les opportunités offertes dans ce contexte par l'enseignement médical, il est indispensable de recueillir les attentes des principaux groupes d'intérêts et d'en tenir compte. Le présent article prend l'exemple de SOS Ärzte de Zurich et se fonde sur une procédure établie dans le domaine de la gestion de projets pour analyser la question de manière systématique. spektive des Weiterbildungsverantwortlichen eine Standortbestimmung notwendig. Hierbei sollten im Kontext des jeweiligen Arbeitsortes folgende Fragen beleuchtet werden:

- Wer verfügt in meiner Institution aktuell über Einfluss und Interesse, die Weiterbildung zu beeinflussen?

- Was sind die Bedürfnisse dieser Interessengruppen bezüglich der Weiterbildung?

Der Begriff «Stakeholder» steht im weiteren Sinn für Personen, Gruppen oder Organisationen, die von einem Projekt beeinflusst werden und/ oder ein Projekt beeinflussen.

- Wie können die bestehenden und geplanten Weiterbildungsprojekte so angepasst werden, dass die Bedürfnisse der wichtigsten Interessengruppen besser berücksichtigt werden?

Im vorliegenden Artikel wird mit der StakeholderAnalyse eine etablierte Methode aus dem Bereich des Projektmanagements vorgestellt, die sich für die Beantwortung der obigen Fragen eignet. Zur Illustration wird die Umsetzung einer Stakeholder-Analyse am Beispiel der Weiterbildung der SOS Ärzte aufgezeigt.

\section{Stakeholder-Analyse, Konzept}

Der Begriff «Stakeholder» steht im weiteren Sinne für Personen, Gruppen oder Organisationen, die von einem Projekt beeinflusst werden und/oder ein Projekt beeinflussen können [4]. Die Stakeholder-Ana- 


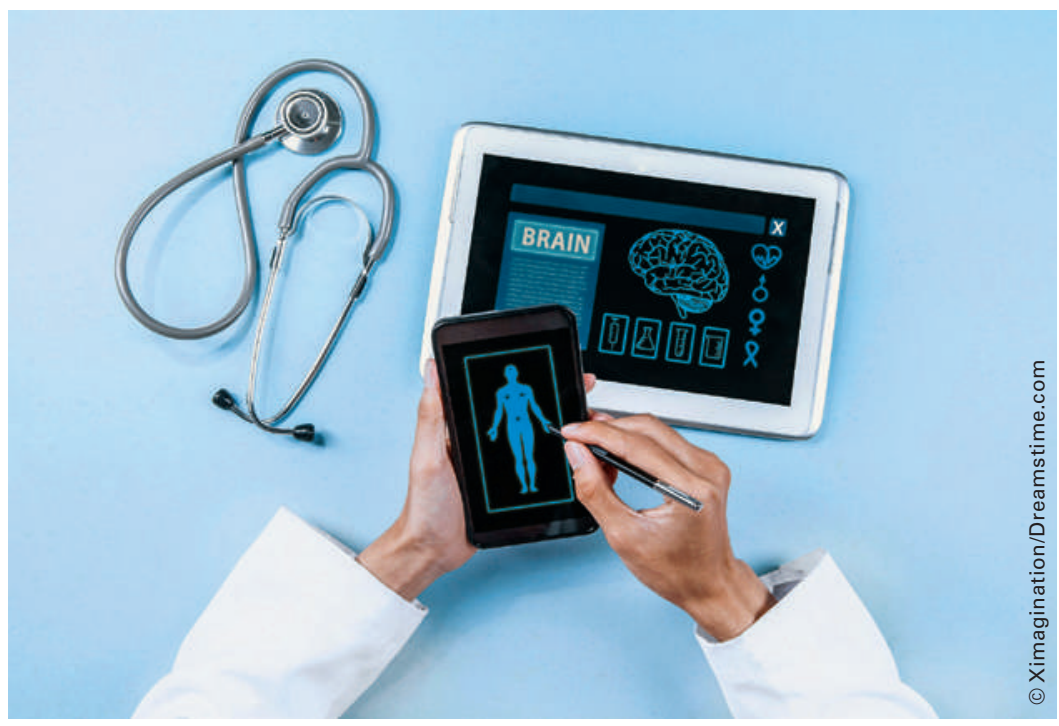

Die Zeit zwischen Patientenbesuchen zur Weiterbildung nutzen - möglich wird es durch einen Zugang zu den Lerninhalten über das Smartphone.

lyse beschreibt eine Methode zur systematische Erfassung der Bedürfnisse wichtiger Stakeholder mit dem Ziel, das Projekt besser nach den Bedürfnissen der Stakeholder auszurichten [4]. Der Wert einer gezielten Stakeholder-Analyse ist in der professionellen Projektbetreuung unbestritten [5, 6]. Untersuchungen zeigen sogar, dass die mangelhafte Erfassung der Interessen wichtiger Stakeholder zu den häufigsten Gründen für das Scheitern von Projekten gehört [7]. Umgesetzt auf die ärztliche Weiterbildung bedeutet dies also, dass im Setting der institutionellen Gegebenheiten die Bedürfnisse der wichtigsten Stakeholder identifiziert und die Weiterbildungsangebote entsprechend bedürfnisgerecht angepasst werden müssen.

\section{Stakeholder in der Weiterbildung der SOS Ärzte}

In der Stakeholder-Analyse konzentrieren sich die Bemühungen auf diejenigen Stakeholder, die einen grossen Einfluss auf das Projekt besitzen und auch ein Interesse haben, diesen Einfluss geltend zu machen [4]. Zur Identifikation der Stakeholder in der Weiterbildung der SOS Ärzte bedarf es einer kurzen Darstellung der institutionellen Rahmenbedingungen:

Die SOS Ärzte Zürich sind ein privatwirtschaftlich geführtes, finanziell unabhängiges Institut für Notfallmedizin im Grossraum Zürich. Aktuell betreuen 28 Ärzte in Weiterbildung oder mit abgeschlossenem Facharzttitel Patienten in einem breiten Spektrum allgemeinmedizinischer Notfallsituationen, dies ausschliesslich auf Hausbesuchen oder durch telefonische Beratung. Aktuell etablierte interne Weiter- bildungsangebote sind das Tutoring-Projekt (eine angepasste Form des Arbeitsplatz-basierten Assessments [8] während zwei Halbtagen pro Jahr), regelmässige telefonische Patientenbesprechungen mit dem Einsatzleiter, Rückmeldungen zu den diktierten Berichten sowie ein breites Angebot an Literatur zum Selbststudium in der Einsatzzentrale. Verschiedene weitere Weiterbildungsformate befinden sich derzeit in der Konzeptions- oder Entwicklungsphase (s.u.). Als wichtigste Stakeholder bezüglich der internen Weiterbildung der SOS Ärzte können die Dienstärzte sowie die ärztliche Leitung mit mutmasslich teilweise unterschiedlichen Bedürfnissen identifiziert werden. Der relative Einfluss der beiden Gruppen variiert je nach finanziellem Aufwand und dem Ausmass der - vom Lernenden geforderten - Eigeninitiative des Weiterbildungsformats. Alle übrigen Stakeholder verfügen über wenig Möglichkeiten respektive wenig Interesse an der direkten Einflussnahme, weshalb sich die weitere Analyse auf die beiden erstgenannten Gruppen beschränkt.

\section{Bedürfnisse der Stakeholder erfassen}

In einem nächsten Schritt werden die Bedürfnisse der Haupt-Stakeholder genauer erfasst, im vorliegenden Fall die Vorstellungen der Dienstärzte sowie der ärztlichen Leitung der SOS Ärzte bezüglich Inhalt,

Die mangelhafte Erfassung der Interessen wichtiger Stakeholder gehört zu den häufigsten Gründen für das Scheitern von Projekten.

Organisation und Endpunkte der Weiterbildung. Die beiden Gruppen wurden mittels Online-Fragebogen $\mathrm{zu}$ folgenden Aspekten befragt: Bestehende (a) und zukünftige (b) Weiterbildungs-Projekte sowie generelle organisatorische Aspekte der Weiterbildung (c). Die eingegangenen Antworten (Ärztliche Leitung: $\mathrm{N}=6 / 6$, Rücklaufquote $100 \%$, Dienstärzte: $N=19 / 22$, Rücklaufquote $86,3 \%$ ) wurden deskriptiv-statistisch ausgewertet und werden nun im folgenden Abschnitt gegenübergestellt.

\section{Bedürfnisse wichtiger Stakeholder bei den SOS Ärzten}

a) Bestehende Weiterbildungsangebote

Die bereits bestehenden Lernangebote werden von den Dienstärzten mehrheitlich positiv eingeschätzt. So wird der Lerneffekt aller bestehenden Angebote zwischen «mässig» und "gross» eingestuft. Am wertvollsten wird dabei die telefonische Rücksprache mit 
dem Einsatzleitenden sowie das Tutoring bewertet. Die schriftlichen Rückmeldungen zu Berichten und das Selbststudium mittels der in der Einsatzzentrale zur Verfügung gestellten Ressourcen werden etwas weniger geschätzt.

b) Zukünftige Weiterbildungsangebote

Die meisten Dienstärzte zeigen sich motiviert, an zusätzlichen Weiterbildungsangeboten teilzunehmen (4,17 auf einer fünfstufigen Likert-Skala).

Bezüglich der Frage, auf welche Fachgebiete sich die Weiterbildung konzentrieren sollte, zeigt sich eine weitgehende Übereinstimmung der Präferenzen von Dienstärzten mit denen der ärztlichen Leitung (Abb. 1). So stehen allgemein- und notfallmedizinische Inhalte im Zentrum. Hervorzuheben ist zudem der hohe Bedarf an psychiatrischer Weiterbildung, Weiterbildungsinhalte in Anästhesie und Gynäkologie/Geburtshilfe hingegen sind weniger gefragt.

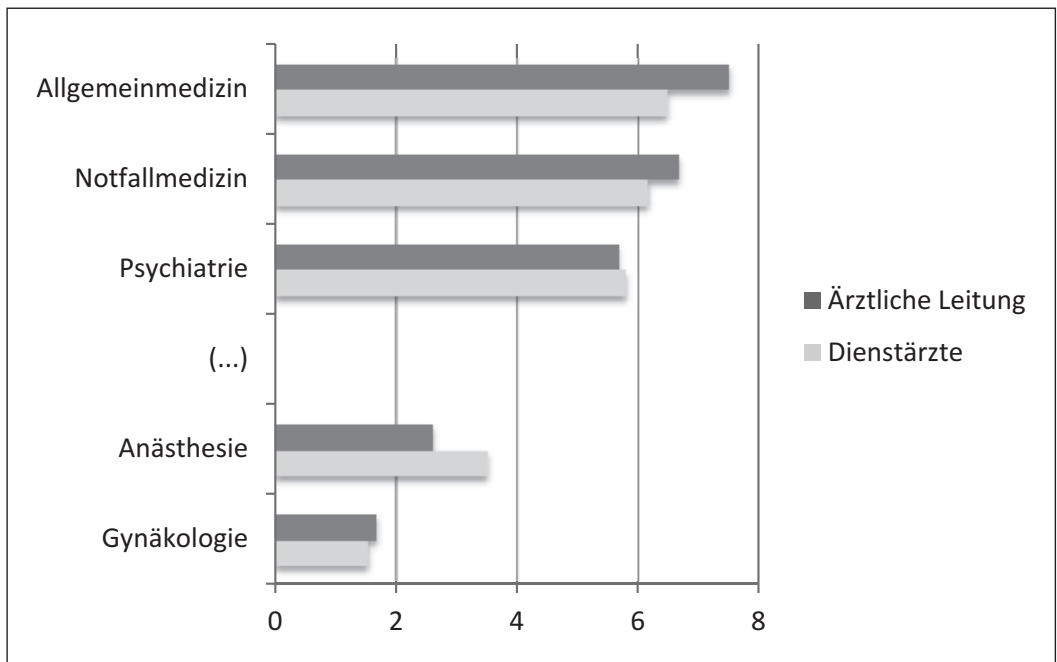

Abbildung 1: Welchen der folgenden fachlichen Themen sollte sich die Weiterbildung der SOS Ärzte widmen? (Erstelle eine hierarchische Liste)

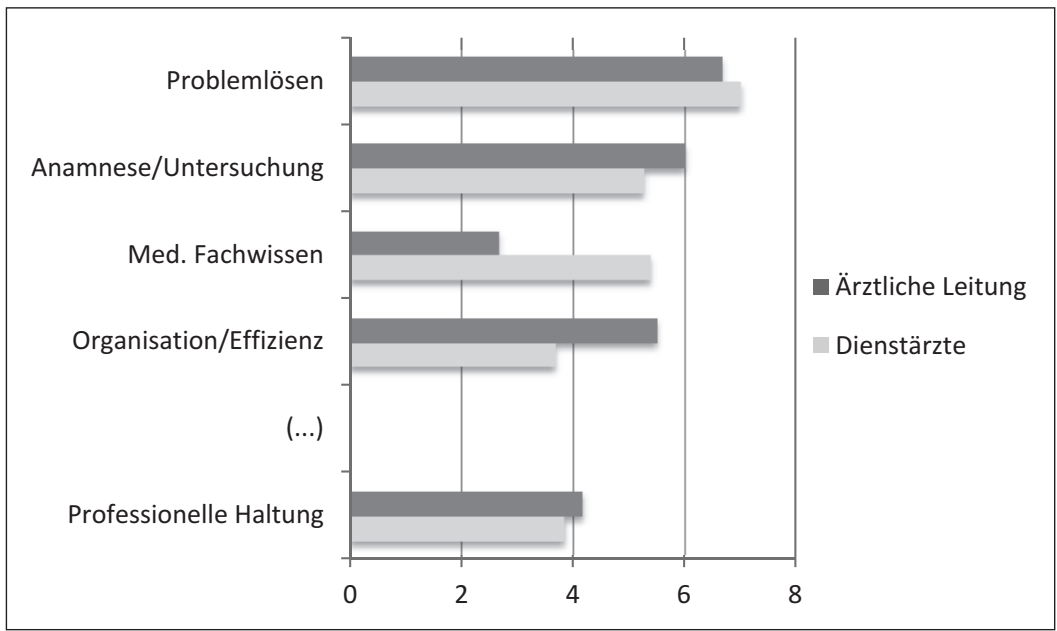

Abbildung 2: Welche Kompetenzen möchtest du (sollen die Dienstärzte) in der Weiterbildung der SOS Ärzte erweitern? (Erstelle eine hierarchische Liste)
Aus kompetenzbasierter Perspektive besteht Weiterbildungsbedarf übereinstimmend für beide Gruppen v.a. im Bereich des Problemlöseverhaltens sowie in der Schärfung klinischer Fähigkeiten (Abb. 2). Für die ärztliche Leitung stellt zudem die effiziente Betriebseinführung für neue Arbeitskräfte ein wichtiges Element dar.

c) Organisation Weiterbildung

Durch das spezielle Setting bei den SOS Ärzten mit dem Schichtbetrieb sowie der meist direkten Weiter fahrt mit dem Dienstwagen von einem Patienten zum nächsten ergeben sich für die Weiterbildung einige Besonderheiten. So geben die Dienstärzte an, während eines durchschnittlichen Dienstes 25 Minuten an ungenutzter Zeit ausserhalb der betriebseigenen Räumlichkeiten zu haben, die sie für Weiterbildung einsetzen möchten, dies in der Regel während des Wartens auf den nächsten Auftrag nach einem abgeschlossenen Patientenbesuch.

Frühere Versuche zeitlich synchroner Weiterbildungs-Veranstaltungen (z.B. Vorlesungscurriculum) waren jeweils an der mangelnden Vereinbarkeit mit dem Dienstplan gescheitert, weshalb aus der Perspektive der ärztlichen Leitung auch die «Kompatibilität mit dem Dienstbetrieb» die wichtigste organisatorische Eigenheit von Weiterbildungsformaten darstellt.

Vonseiten der Dienstärzte werden Weiterbildungsangebote gewünscht, die inhaltlich für die tägliche Arbeit relevant sowie innerhalb der normalen Dienstzeiten absolvierbar sind und zudem einen Austausch mit anderen Ärzten beinhalten. Die Frage nach der Vorliebe für verschiedene Lernformen ergibt keine eindeutige Präferenz.

\section{Projektanpassung auf Basis der erfassten Bedürfnisse}

Im letzten Schritt einer Stakeholder-Analyse werden die in der Befragung identifizierten Bedürfnisse durch konkrete Änderungen des Projektinhalts, hier der Form und des Inhalts der bestehenden oder geplanten Weiterbildungsprojekte, umgesetzt. Im folgenden Abschnitt werden Projektanpassungen oder strategische Entscheidungen beschrieben, die auf Basis der Erkenntnisse der Stakeholder-Analyse im Weiterbildungsprogramm der sos Ärzte getätigt wurden.

Die vorliegende Befragung zeigt, dass die bestehenden Weiterbildungsangebote der SOS Ärzte geschätzt werden. Diese werden daher weitergeführt. Hinsichtlich neuer Weiterbildungsprojekte wird aufgrund der Resultate folgende Priorisierung vorgenommen: 
Die Idee regelmässiger Fallbesprechungen in synchroner Form wird verworfen: Regelmässige Treffen scheinen mit einem zu hohen zeitlichen und organisatorischen Aufwand verbunden zu sein. Eine angedachte Zusammenarbeit mit dem Simulationszentrum der Anästhesie des USZ wird aktuell ebenfalls zurückgestellt. Dies, da kein ausgeprägtes Bedürfnis nach dieser aufwendigen Form der Weiterbildung zu bestehen scheint.

\section{Personalisierte Logins gewährleisten die Verfügbarkeit unterwegs von betriebs- eigenen Dienst-Smartphones.}

Die in der Stakeholderanalyse erfassten Bedürfnisse lassen sich unter den bestehenden Rahmenbedingungen stattdessen am besten im Rahmen einer virtuellen Lernplattform erfüllen; auf deren Entwicklung konzentrieren sich daher aktuell die Ressourcen. Die Vorteile einer webbasierten Lernumgebung, die auf Basis der Open Source Software «Moodle» eingerichtet werden soll, bietet mannigfaltige Vorteile:

- Personalisierte Logins gewährleisten die Verfügbarkeit unterwegs von den betriebseigenen DienstSmartphones sowie von den Tablets in der Einsatzzentrale.

- Virtuelle Fallbesprechungen mit echten, direkt aus dem betriebseigenen System extrahierten Berichten gewährleisten eine hohe Interaktivität im asynchronen, zeitlich unabhängigen Modus sowie ein angewandtes, problemorientiertes Lernen.

- Ein News-Forum kommt dem Bedürfnis der ärztlichen Leitung nach Verbesserung der internen Kommunikation entgegen.

- Der Zugang zu Lerninhalten ist jederzeit unterwegs vom Dienst-Smartphone aus möglich. Die zwischen zwei Patientenbesuchen brachliegenden zeitlichen Ressourcen können so für die Weiterbildung genutzt werden.

Dr. med. Roman Hari

SOS Ärzte

Weinbergstrasse 68

CH-8006 Zürich

roman.hari[at]sos-aerzte.com

www.sos-aerzte.com

\section{Schlussfolgerung}

Wie die konkrete Anwendung zeigt, können mittels geeigneter Erfassung von Bedürfnissen der wichtigsten Stakeholder wertvolle Erkenntnisse für die weitere Gestaltung der Weiterbildung gewonnen werden. Projekte werden so bedarfsgerechter gestaltet und die vorhandenen Ressourcen bei simultanen Teilprojekten gebündelt.

Das beschriebene Vorgehen hat jedoch auch wesentliche Einschränkungen:

- Aus der berufsethischen Perspektive ist die alleinige Ausrichtung der Weiterbildungsinhalte auf die Bedürfnisse des ärztlichen Personals sowie der betriebswirtschaftlichen Bedürfnisse der Weiterbildungsstätte fragwürdig: Die ärztliche Weiterbildung muss in letzter Konsequenz die verbesserte Versorgung des Patienten zum Ziel haben. Sie sollte sich also auch auf Gebiete fokussieren, wo objektiv messbare sowie subjektiv von Patienten empfundene Defizite in der ärztlichen Leistung bestehen. Dieser Notwendigkeit wird jedoch durch die bestehenden rechtlichen Rahmenbedingungen (Weiterbildungsordnung FMH, Facharztprüfungen) auch bereits Rechnung getragen.

- Der empirische Nachweis, dass der Einsatz von Strategien aus dem Projektmanagement die Chancen von Projekten im Allgemeinen erhöht, ist hinreichend erbracht (s.o.). Ob dies auch auf das Gelingen von Weiterbildungsprojekten in der Medizin übertragbar ist, verlangt weiterer Erforschung.

Zusammenfassend ist die gezielte Erfragung von Bedürfnissen wichtiger Stakeholder einer der Kernpunkte in der Planung oder Überarbeitung eines Weiterbildungscurriculums. Um die grosse Chance, die sich der ärztlichen Weiterbildung aktuell durch den Ärztemangel bietet, nicht zu verpassen, ist die ausgewogene Berücksichtigung dieser Bedürfnisse entscheidend.

Interessenkonflikt

Der Auor ist als Projektleiter Weiterbildung bei den SOS Ärzten angestellt. 


\section{Literatur}

1 Schweizerischer Bundesrat (Hrsg). Strategie gegen Ärztemangel und zur Förderung der Hausarztmedizin [Internet]. www.bag. admin.ch/themen/berufe/13932/13933/14201/index. html?lang=de. 25.02 .2015

2 Junior Doc Medical Team (Hrsg). Staatsabgänger Studie 2013/2014 [Internet]. www.jdmt.ch/index.php?id=1648 (Artikel erhältlich über office[at]jdmt.ch). 25.2.2015.

3 Schweizerisches Institut für ärztliche Weiter- und Fortbildung SIWF (Hrsg). Weiterbildungsordnung (WBO) 21. Juni 2000. Letzte Revision: 4. September 2014 [Internet]. http://fmh.ch/ bildung-siwf/weiterbildung/fuer-leiter-wb-staetten/ weiterbildungsstaetten/facharzttitel.html. 25.2.2015.

4 Project Management Institute (Hrsg.). A Guide to the Project Management Body of Knowledge (PMBOK ${ }^{\circledR}$ Guide). Fifth edition. Pennsylvania: PMI Inc; 2013. S. 391-415.
5 Varvasovszky Z, Brugha R. How to Do (or Not to Do) ... A Stakeholder Analysis. Health Policy Planning. 2000;15(3):338-45.

6 Achterkamp MC, Vos JFC. Investigating the Use of the Stakeholder Notion in Project Management Literature, a Meta-Analysis. International Journal of Project Management. 2008;26:749-57.

7 Goatham R, Henry B, Ryder G. Why Projects Fail. A Resource for Advanced Project Management and Organizational Learning Focused on Improving Project Success Rates [Internet]. http://calleam.com/WTPF/. 25.2.2015.

8 Rogausch A, Berendonk C, Giger M, Bauer W, Beyeler C. Ziel und Nutzen des Arbeitsplatz-basierten Assessments im klinischen Alltag. Schweiz Med Forum. 2012;12(10):214-17. 